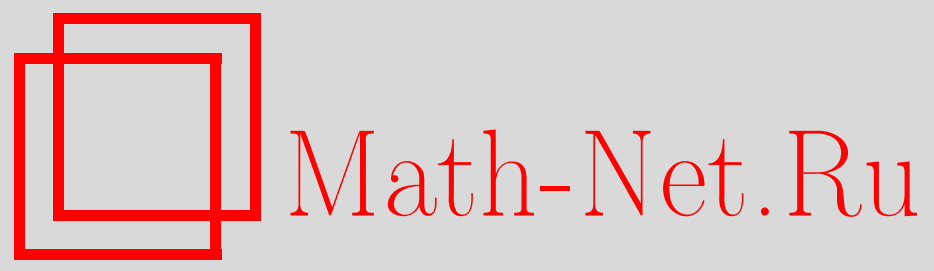

Х. Г. Умаров, Явный вид решения задачи Коши для линеаризованной системы уравнений фазового поля, Вестн. Сам. гос. техн. ун-та. Сер. Физ.-мат. науки, 2012, выпуск 1(), 256-260

DOI: https://doi.org/10.14498/vsgtu977

Использование Общероссийского математического портала Math-Net.Ru подразумевает, что вы прочитали и согласны с пользовательским соглашением

http: //www . mathnet.ru/rus/agreement

Параметры загрузки:

IP : 54.197 .130 .99

26 апреля 2023 г., 12:36:58

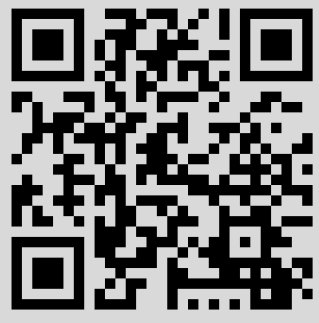


УДК 517.95+517.986.7

\section{ЯВНЫЙ ВИД РЕШЕНИЯ ЗАДАЧИ КОШИ ДЛЯ ЛИНЕАРИЗОВАННОЙ СИСТЕМЫ УРАВНЕНИЙ ФАЗОВОГО ПОЛЯ}

\section{Х. Г. Умаров}

Чеченский государственный университет, 364907, Россия, Грозный, ул. Шерипова, 32.

E-mail: umarov50@mail.ru

Для линеаризованной системы уравнений фазового поля получен явный вид решения задачи Коши сведением ё̈ к абстрактной задаче Коши в банаховом пространстве.

Ключевые слова: линеаризованная система уравнений фазового поля, сильно непрерывные полугруппы операторов в банаховых пространствах.

Рассмотрим в области $(x, y, z) \in \mathbb{R}^{3}, t>0$, задачу Коши для системы уравнений в частных производных:

$$
\begin{gathered}
\left\{\begin{array}{c}
v_{t}=\Delta v-\triangle w, \\
\triangle w+(\alpha-1) w+v=0,
\end{array}\right. \\
\left.v\right|_{t=0}=\varphi(x, y, z),
\end{gathered}
$$

которая является, с точностью до линейной замены, линеаризацией в нуле квазистационарной системы уравнений фазового поля [1, с. 24], моделирующей в рамках мезоскопической теории фазовые переходы первого рода. В системе (1) искомые функции $v=v(x, y, z, t), w=w(x, y, z, t)$ характеризуют распределение температуры и параметр порядка; $\triangle=\partial^{2} / \partial x^{2}+\partial^{2} / \partial y^{2}+\partial^{2} / \partial z^{2}$ - дифференциальный оператор Лапласа в $\mathbb{R}^{3} ; \alpha$-постоянная, зависящая от характеристик среды.

В работе [1] система (1) исследуется в ограниченной области методами теории уравнений соболевского типа с относительно секториальными операторами.

Наша цель - получить для системы (1) явный вид решения задачи Коши в $\mathbb{R}^{3}$. Явный вид решения задачи Коши (первой начально-краевой задачи в полупространстве и смешанной задачи в пространственном слое в предположении анизотропии среды) получен в [2].

Будем искать решение системы $(1)$ в банаховом пространстве $L_{p}\left(\mathbb{R}^{3}\right), 1<p<+\infty$. В этом пространстве оператор Лапласа с областью определения $\mathcal{D}(\triangle)=\left\{\psi \in L_{p}\left(\mathbb{R}^{3}\right)\right.$ : $\left.\triangle \psi \in L_{p}\left(\mathbb{R}^{3}\right)\right\}$ является производящим оператором сжимающей сильно непрерывной (более того - аналитической) полугруппы $U(t ; \triangle)$ класса $C_{0}[3$, с. $228 ; 4$, с. 58$]$ :

$$
U(t ; \triangle) \psi(x, y, z)=(4 \pi t)^{-3 / 2} \iiint_{\mathbb{R}^{3}} e^{-\left((x-\xi)^{2}+(y-\eta)^{2}+(z-\zeta)^{2}\right) /(4 t)} \psi(\xi, \eta, \zeta) d \xi d \eta d \zeta
$$

и его область определения является пространством Соболева: $\mathcal{D}(\triangle)=W_{p}^{2}\left(\mathbb{R}^{3}\right)$.

Исключим из системы (1) одну из неизвестных функций. Для этого воспользуемся тем, что положительная полуось принадлежит резольвентному множеству оператора Лапласа в пространстве $L_{p}\left(\mathbb{R}^{3}\right):$ предполагая, что $\alpha<1$, из второго уравнения системы (1) выводим

$$
w=((1-\alpha) I-\triangle)^{-1} v
$$

Хасан Галсанович Умаров (к.ф.-м.н., доц.), доцент, каф. дифференциальных уравнений. 
Подставляя полученное значение $w$ в первое уравнение системы $(1)$, имеем

$$
v_{t}=\Delta v+v-(1-\alpha)((1-\alpha) I-\triangle)^{-1} v .
$$

Таким образом, обозначая в уравнении (5) операторы $\triangle=A$ с областью определения $\mathcal{D}(A)=W_{p}^{2}\left(\mathbb{R}^{3}\right)$ и $I-(1-\alpha)((1-\alpha) I-\triangle)^{-1}=B$ с областью определения $\mathcal{D}(B)=L_{p}\left(\mathbb{R}^{3}\right)$, приходим к абстрактному дифференциальному уравнению первого порядка в банаховом пространстве $L_{p}\left(\mathbb{R}^{3}\right)$ :

$$
v_{t}=(A+B) V
$$

относительно функции $V=V(t):[0,+\infty) \stackrel{V}{\rightarrow} L_{p}\left(\mathbb{R}^{3}\right)$ по правилу: для любого $t \geqslant 0$ $V(t)=v(x, y, z, t) \in L_{p}\left(\mathbb{R}^{3}\right)$. Начальным условием для уравнения (6) будет являться равенство

$$
\left.V\right|_{t=0}=\phi
$$

где $\phi=V(0)=v(x, y, z, 0)=\varphi(x, y, z)$ - заданный элемент пространства $L_{p}\left(\mathbb{R}^{3}\right)$ в соответствии с условием $(2)$.

Оператор $B=I-(1-\alpha)((1-\alpha) I-\triangle)^{-1}-$ линеен и ограничен на всём пространстве $L_{p}\left(\mathbb{R}^{3}\right)$, поэтому является производящим оператором сильно непрерывной полугруппы (более того - группы) класса $C_{0}: U(t ; B) \psi=\sum_{k=0}^{+\infty} \frac{t^{k}}{k !} B^{k} \psi$, для которой справедливо представление

$$
U(t ; B) \psi=e^{t} \sum_{k=0}^{+\infty} \frac{(-1)^{k} t^{k}(1-\alpha)^{k}}{k !}((1-\alpha) I-\triangle)^{-k} \psi,
$$

где $\psi$ - произвольный элемент пространства $L_{p}\left(\mathbb{R}^{3}\right)$. Для полугруппы $U(t ; B), t \geqslant 0$, справедлива оценка нормы:

$$
\|U(t ; B)\| \leqslant e^{t} \sum_{k=0}^{+\infty} \frac{(1-\alpha)^{k} t^{k}}{k !}\left\|((1-\alpha) I-\triangle)^{-1}\right\|^{k} \leqslant e^{2 t} .
$$

Выражая степени резольвенты $((1-\alpha) I-\triangle)^{-k}$ через полугруппу (3), находим представление полугруппы, порождаемой оператором $B$ :

$$
U(t ; B) \psi=e^{t}\left[\psi-\sqrt{(1-\alpha) t} \int_{0}^{+\infty} e^{-(1-\alpha) s} J_{1}(2 \sqrt{(1-\alpha) t s}) U(s ; \triangle) \psi \frac{d s}{\sqrt{s}}\right],
$$

где $J_{1}(z)=\sum_{k=0}^{+\infty} \frac{(-1)^{k}(z / 2)^{2 k+1}}{k !(k+1) !}-$ функция Бесселя.

Из полученных представлений полугрупп $U(t ; A)$ и $U(t ; B)$ через полугруппу, порождаемую оператором Лапласа, следует их коммутирование.

При возмущении производящего оператора $A$ сильно непрерывной полугруппы $U(t ; A)$ класса $C_{0}$ линейным ограниченным оператором $B$ оператор $A+B$ с областью определения $\mathcal{D}(A+B)=\mathcal{D}(A)$ также порождает [5, с. 403] сильно непрерывную полугруппу $U(t ; A+B)$ класса $C_{0}$, при этом возмущённая полугруппа определяется разложением в ряд:

$$
U(t ; A+B) \psi=\sum_{k=0}^{+\infty} U_{k}(t) \psi, \quad t \geqslant 0
$$

где

$$
U_{k}(t) \psi=\int_{0}^{t} U(t-s ; A) B U_{k-1}(s) \psi d s, \quad k \geqslant 1
$$


и $U_{0}(t) \psi=U(t ; A) \psi$ для произвольного элемента $\psi$ банахова пространства, причём ряд абсолютно сходится равномерно по $t$ в любом конечном интервале положительной полуоси.

В нашем случае возмущающий линейный ограниченный оператор $B$ коммутирует с полугруппой, порождаемой возмущаемым оператором $A: B U(t ; A) \psi=U(t ; A) B \psi$, так как этим свойством обладают резольвента $(\lambda I-A)^{-1}$ и полугруппа $U(t ; A)$ для любого производящего оператора $A$ сильно непрерывной полугруппы класса $C_{0}$. Отсюда следует, что

$$
U_{k}(t) \psi=\frac{t^{k}}{k !} B^{k} U(t ; A) \psi=\frac{t^{k}}{k !} U(t ; A) B^{k} \psi
$$

и, значит, справедливо следующее представление для полугруппы, порождаемой оператором $A+B$ :

$$
\begin{aligned}
& U(t ; A+B) \psi=U(t ; A) U(t ; B) \psi=U(t ; B) U(t ; A) \psi= \\
& =e^{t}\left[U(t ; \triangle) \psi-\sqrt{(1-\alpha) t} \int_{0}^{+\infty} e^{-(1-\alpha) s} J_{1}(2 \sqrt{(1-\alpha) t s}) U(s+t ; \triangle) \psi \frac{d s}{\sqrt{s}}\right],
\end{aligned}
$$

и оценка нормы

$$
\|U(t ; A+B)\| \leqslant\|U(t ; A)\| \cdot\|U(t ; B)\| \leqslant e^{2 t} .
$$

Для того чтобы задача Коши (6), (7) была равномерно корректной [4, с. 64], необходимо и достаточно, чтобы оператор $A+B$ был производящим оператором полугруппы класса $C_{0}$, при этом решение задачи Коши $(6),(7)$ даётся формулой

$$
V=U(t ; A+B) \phi
$$

если начальное данное $\varphi$ принадлежит области определения $\mathcal{D}(A+B)$.

Принадлежность элемента $\varphi$ множеству $\mathcal{D}(A+B)$ будет следовать из принадлежности начального данного $\varphi=\varphi(x, y, z)$ пространству Соболева $W_{p}^{2}\left(\mathbb{R}^{3}\right)$. Предполагая это выполненным и используя представление (8), выводим из (10) формулу решения задачи Коши для уравнения (5):

$$
\begin{aligned}
v(x, y, z, t) & =e^{t}[U(t ; \triangle) \varphi(x, y, z)- \\
& \left.-\sqrt{(1-\alpha) t} \int_{0}^{+\infty} e^{-(1-\alpha) s} J_{1}(2 \sqrt{(1-\alpha) t s}) U(s+t ; \triangle) \varphi(x, y, z) \frac{d s}{\sqrt{s}}\right],
\end{aligned}
$$

для которого, в силу (9), справедлива оценка

$$
\|v(x, y, z, t)\|_{L_{p}\left(\mathbb{R}^{3}\right)} \leqslant e^{2 t}\|\varphi(x, y, z)\|_{L_{p}\left(\mathbb{R}^{3}\right)} .
$$

Наконец, используя в формуле (11) представление (3) полугруппы, порождаемой оператором Лапласа, получаем явный вид решения задачи Коши (5), (2):

$$
\begin{gathered}
v(x, y, z, t)=\frac{1}{\pi \sqrt{\pi}}\left[\iiint_{\mathbb{R}^{3}} e^{-\xi^{2}-\eta^{2}-\zeta^{2}} \varphi(x+2 \sqrt{t} \xi, y+2 \sqrt{t} \eta, z+2 \sqrt{t} \zeta) d \xi d \eta d \zeta-\right. \\
-\sqrt{(1-\alpha) t} \int_{0}^{+\infty} e^{-(1-\alpha) s} J_{1}(2 \sqrt{(1-\alpha) t s}) \frac{d s}{\sqrt{s}} \times \\
\left.\times \iiint_{\mathbb{R}^{3}} e^{-\xi^{2}-\eta^{2}-\zeta^{2}} \varphi(x+2 \sqrt{s+t} \xi, y+2 \sqrt{s+t} \eta, z+2 \sqrt{s+t} \zeta) d \xi d \eta d \zeta\right]
\end{gathered}
$$


Теперь из соотношения (4), используя формулу и оценку резольвенты оператора Лапласа в $L_{p}\left(\mathbb{R}^{3}\right)$, получаем явный вид второй искомой функции системы $(1)$ :

$$
\begin{aligned}
& \begin{array}{l}
w(x, y, z, t)=\frac{1}{\pi \sqrt{\pi}} e^{t}\left[\int_{0}^{+\infty} e^{-(1-\alpha) r} d r \times\right. \\
\times \iiint_{\mathbb{R}^{3}} e^{-\xi^{2}-\eta^{2}-\zeta^{2}} \varphi(x+2 \sqrt{r+t} \xi, y+2 \sqrt{r+t} \eta, z+2 \sqrt{r+t} \zeta) d \xi d \eta d \zeta- \\
\quad-\sqrt{(1-\alpha) t} \int_{0}^{+\infty} e^{-(1-\alpha) r} d r \int_{0}^{+\infty} e^{-(1-\alpha) s} J_{1}(2 \sqrt{(1-\alpha) t s}) \frac{d s}{\sqrt{s}} \times \\
\left.\times \iiint_{\mathbb{R}^{3}} e^{-\xi^{2}-\eta^{2}-\zeta^{2}} \varphi(x+2 \sqrt{r+s+t} \xi, y+2 \sqrt{r+s+t} \eta, z+2 \sqrt{r+s+t} \zeta) d \xi d \eta d \zeta\right]
\end{array}
\end{aligned}
$$

и её оценку

$$
\|w(x, y, z, t)\|_{L_{p}\left(\mathbb{R}^{3}\right)} \leqslant \frac{e^{2 t}}{1-\alpha}\|\varphi(x, y, z)\|_{L_{p}\left(\mathbb{R}^{3}\right)}
$$

\section{Таким образом, имеет место}

ТЕорема. Предположим, что в системе уравнений фазового поля (1) параметр $\alpha$ удовлетворяет неравенству $\alpha<1$ и пусть в задаче Коши (1), (2) решение $v(x, y, z, t), w(x, y, z, t)$ ищется в пространстве $L_{p}\left(\mathbb{R}^{3}\right), 1<p<+\infty$, а начальное данное $\varphi(x, y, z)$ принадлежит пространству Соболева $W_{p}^{2}\left(\mathbb{R}^{3}\right)$, тогда единственное решение этой системы даётся формулами (13), (14) и для него справедливь оценки (12), (15).

ЗАмечАниЕ. Отметим, что по переменной $t$ решение (13) удовлетворяет полугрупповому свойству, дифференцируемо при $t \geqslant 0$ и бесконечно дифференцируемо при $t>0$. По переменным $(x, y, z)$ значения решения при $t \geqslant 0$ принадлежат пространству Соболева $W_{p}^{2}\left(\mathbb{R}^{3}\right)$ и, значит, у решения существуют частные и смешанные обобщённые производные по переменным $(x, y, z)$ до второго порядка включительно, принадлежащие пространству $L_{p}\left(\mathbb{R}^{3}\right)$ при $t \geqslant 0$, и обобщённые производные любого порядка при $t>0$. Из оценок (12), (15) следует непрерывная зависимость решения системы (1) от начального данного на любом конечном временном отрезке.

\section{БИБЛИОГРАФИЧЕСКИЙ СПИСОК}

1. Загребина С. А. О задаче Шоуолтера-Сидорова // Изв. вузов. Матем., 2007. № 3. С. 2228; англ. пер.: Zagrebina S. A. On the Showalter-Sidorov problem // Russian Math. (Iz. VUZ), 2007. Vol.51, no. 3. Pp. 19-24.

2. Умаров X. Г. Явный вид решения линеаризованной системы уравнений фазового поля. Грозный: ЧечГУ, 2010. 27 с. (Деп. в ВИНИТИ 24.05.10 № 303-В2010) [Umarov Kh. G. The explicit form of solutions for linearized system of phase field equations. Groznyi: ChechGU, 2010. 27 pp. (Deposited at VINITI 24.05.10 No. 303-B2010)]

3. Трибель X. Теория интерполяции, функциональные пространства, дифференциальные операторы. М.: Мир, 1980. 664 с. [Tribel' Kh. Interpolation theory, function spaces, differential operators. Moscow: Mir, 1980. 664 pp.]

4. Крейн С.Г. Линейные дифференциальные уравнения в банаховом пространстве. М.: Наука, 1967. 464 c. [Krein S. G. Linear differential equations in a Banach space. Moscow: Nauka, 1967. 464 pp.] 
5. Хилле Э., Филлипс Р. С. Функциональный анализ и полугруппы. М.: Иностр. лит., 1962. 829 c. [Hille E., Fillips R. Functional analysis and semi-groups. Moscow: Inostran. Lit., 1962. 829 pp.]

Поступила в редакцию $30 / \mathrm{VI} / 2011$;

в окончательном варианте - 29/XI/2011.

MSC: 35G10; 47D06

EXPLICIT SOLUTION OF CAUCHY PROBLEM FOR THE LINEARIZED SYSTEM OF PHASE FIELD EQUATIONS

\section{Kh. G. Umarov}

Chechen State University,

32, Sheripova st., Groznyi, 364907, Russia.

E-mail: umarov50@mail.ru

The explicit solution of Cauchy problem for the linearized system of phase field equations is received by reduction it to the abstract Cauchy problem in Banach space.

Key words: linearized system of phase field equations, strongly continuous semi-groups of operators in Banach spaces.

Original article submitted 30/VI/2011; revision submitted $29 / \mathrm{XI} / 2011$.

Khasan G. Umarov (Ph. D. (Phys. \& Math.)), Associate Professor, Dept. of Differential Equations. 